University of Wollongong

Research Online

Faculty of Engineering - Papers (Archive)

Faculty of Engineering and Information

Sciences

2003

\title{
MOSFET dosimetry for microbeam radiation therapy at the European Synchrotron Radiation Facility
}

\author{
E. Brauer-Krisch \\ European Synchrotron Radiation Facility, France
}

A. Bravin

European Synchrotron Radiation Facility, France

M. L. Lerch

University of Wollongong, mlerch@uow.edu.au

Anatoly B. Rosenfeld

University of Wollongong, anatoly@uow.edu.au

J. Stepanek

Paul Scherrer Institute, Switzerland

See next page for additional authors

Follow this and additional works at: https://ro.uow.edu.au/engpapers

Part of the Engineering Commons

https://ro.uow.edu.au/engpapers/149

\section{Recommended Citation}

Brauer-Krisch, E.; Bravin, A.; Lerch, M. L.; Rosenfeld, Anatoly B.; Stepanek, J.; Di Michiel, M.; and Laissue, J. A.: MOSFET dosimetry for microbeam radiation therapy at the European Synchrotron Radiation Facility 2003.

https://ro.uow.edu.au/engpapers/149

Research Online is the open access institutional repository for the University of Wollongong. For further information contact the UOW Library: research-pubs@uow.edu.au 


\section{Authors}

E. Brauer-Krisch, A. Bravin, M. L. Lerch, Anatoly B. Rosenfeld, J. Stepanek, M. Di Michiel, and J. A. Laissue 


\title{
MOSFET dosimetry for microbeam radiation therapy at the European Synchrotron Radiation Facility
}

\author{
E. Bräuer-Krisch and A. Bravin \\ European Synchrotron Radiation Facility, B.P. 220, 38043 Grenoble, France \\ M. Lerch and A. Rosenfeld \\ Center for Medical Radiation Physics, University of Wollongong, Wollongong, NSW, 2522, Australia \\ J. Stepanek \\ Paul Scherrer Institut (PSI), CH-5232 Villigen, Switzerland \\ M. Di Michiel \\ European Synchrotron Radiation Facility, B.P. 220, 38043 Grenoble, France \\ J. A. Laissue \\ Institute of Pathology, University of Bern, Murtenstrasse 31, 3010 Bern, Switzerland
}

(Received 13 December 2001; accepted for publication 29 January 2003; published 25 March

2003)

Preclinical experiments are carried out with $\sim 20-30 \mu \mathrm{m}$ wide, $\sim 10 \mathrm{~mm}$ high parallel microbeams of hard, broad-"white"-spectrum $\mathrm{x}$ rays $(\sim 50-600 \mathrm{keV})$ to investigate microbeam radiation therapy (MRT) of brain tumors in infants for whom other kinds of radiotherapy are inadequate and/or unsafe. Novel physical microdosimetry (implemented with MOSFET chips in the "edge-on" mode) and Monte Carlo computer-simulated dosimetry are described here for selected points in the peak and valley regions of a microbeam-irradiated tissue-equivalent phantom. Such microbeam irradiation causes minimal damage to normal tissues, possible because of rapid repair of their microscopic lesions. Radiation damage from an array of parallel microbeams tends to correlate with the range of peak-valley dose ratios (PVDR). This paper summarizes comparisons of our dosimetric MOSFET measurements with Monte Carlo calculations. Peak doses at depths $<22 \mathrm{~mm}$ are $18 \%$ less than Monte Carlo values, whereas those depths $>22 \mathrm{~mm}$ and valley doses at all depths investigated $(2 \mathrm{~mm}-62 \mathrm{~mm})$ are within $2-13 \%$ of the Monte Carlo values. These results lend credence to the use of MOSFET detector systems in edge-on mode for microplanar irradiation dosimetry. () 2003 American Association of Physicists in Medicine. [DOI: 10.1118/1.1562169]

Key words: microdosimetry, microbeam radiation therapy, edge-on MOSFET, synchrotron radiation

\section{INTRODUCTION}

During the past decade, potential applications of microbeam radiation therapy (MRT) have been studied experimentally at the National Synchrotron Light Source (NSLS) at Upton, New York, USA ${ }^{1-3}$ and at the European Synchrotron Radiation Facility (ESRF) in Grenoble, France. ${ }^{4-7}$ For these MRT trials a synchrotron source with extremely high dose rate is needed to irradiate tissues with, for example, $300 \mathrm{~Gy}$, in a fraction of a second, which assures minimal broadening of the microslices of intensely irradiated tissues attributable to movement of the specimen. The preclinical MRT program comprises irradiation of normal brain tissues in weanling piglets $(<7 \mathrm{~kg})$ and suckling rats at the ESRF followed by long-term (9-15 months) neurobehavioral observation, noninvasive imaging and histopathological study. Results 4, 6, 7 show that tissue tolerance to multiple microbeams is surprisingly high, especially for these young animals. MRT might palliate or even ablate malignant brain tumors if there were sufficient sparing of normal tissue radiation damage in the "valleys" between individual microbeams. When such sparing is observed experimentally, knowing the maximum val- ley doses in normal, radiosensitive tissues of the target zones is of crucial importance in predicting what doses and microbeam array geometries are safe and therapeutically useful in clinical research.

The aim of this study was to relate physical peak-tovalley dose ratios with Monte Carlo dose simulations for the geometry used in the experiments. The theoretical calculations, the experimental measurements and the comparison of the data obtained by both methods were all done for a 1 liter cubic PMMA phantom. This phantom has physical properties similar to those of an infant's hindbrain and the hindbrain of the weanling $(\sim 7 \mathrm{~kg})$ piglet, one of our animal models of normal tissue tolerance for this prospective application of MRT. $^{7}$

\section{THEORY}

Most of the theoretical calculations using Monte Carlo $\operatorname{codes}^{8,9}$ have predicted the dose distribution around one single microbeam in the radial direction for very small voxel sizes like $1 \mu \mathrm{m}$ cube. These calculations must be performed for distances from the microbeam peak extending perpen- 


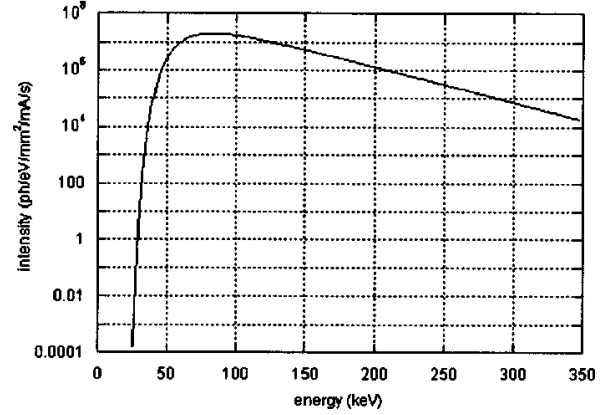

FIG. 1. Measured photon spectrum used for MRT at the ESRF.

dicular about 25 millimeters outwards, since the actual valley dose is calculated by overlapping the tails of the microbeams.

The energy deposition within a $1 \mu \mathrm{m}$ cube can be calculated using sophisticated Monte Carlo programs for the peak and the valley area of the microbeams interacting with a water or plastic phantom. There are several reports on Monte Carlo based computational MRT microdosimetry, 8,9 that quantify the dependence of doses in the peaks and valleys in an $\mathrm{H}_{2} \mathrm{O}$ filled phantom on parameters that include beam photon energy spectrum, size and number of microbeams, dimensions of the phantom, microbeam spacing, position of microbeams, and depth of the point of interest from the phantom surface. Our MOSFET results are compared with computations using the PSI version of the GEANT Monte Carlo photon-electron transport code, ${ }^{10}$ into which the photon and electron cross section and atomic data compiled at Lawrence Livermore National Laboratory in the 1990s have been incorporated (the Evaluated Photon Data Library, EPDL, ${ }^{11,12}$ the Evaluated Electron Data Library EEDL, ${ }^{13}$ and the Evaluated Atomic Data Library EADL ${ }^{14}$ ). The code uses the currently most advanced atomic databases as well as "single-collision" electron transport rather than the "condensed-history" electron transport used in other codes, such as EGS4.0. ${ }^{15}$ The former is an especially important tool for a proper electron tracking in micrometer-sized targets.

The initial filtered photon spectrum and intensity of the wiggler beam can be calculated accurately with various programs, such as XOP. ${ }^{16-18}$ Figure 1 is the photon spectrum at the wiggler beamline ID17 of the ESRF, measured with a powder diffraction method. ${ }^{19}$ This spectrum is read in as input file for the Monte Carlo calculations in the PSI version of the GEANT code. The simulation includes the correct number of microplanar beams assuming all of them to be $25 \mu \mathrm{m}$ thick, which corresponds only to the mean value of our actual microbeams. This inadequate modeling will be corrected in future studies, since the entry of the correct FWHM of each individual microbeam requires a lot of effort and the associated error is insignificant. The dose deposition in the PMMA phantom has been calculated for its correct chemical composition and dimensions as well as the exact points in depth, but without taking into account the Si MOSFET chip, which has been estimated to introduce errors up to $10 \%$. We are currently working on improvement for future studies.
For the MRT irradiation the white synchrotron wiggler spectrum is filtered which results in a spectrum extending from about 50 to well above $350 \mathrm{keV}$ with a maximum at 83 $\mathrm{keV}$. The total number of photons after the filters is roughly $2.7 \times 10^{9}$ photons $\mathrm{s}^{-1} \mathrm{~mm}^{-2} \mathrm{~mA}^{-1}$ in the center of the wiggler radiation cone, which, according to our Monte Carlo computation, imparts an absorbed dose rate at $3 \mathrm{~mm}$ depth in PMMA of $71.5 \mathrm{~Gy} \mathrm{~s}^{-1} \mathrm{~mA}^{-1}$. Also according to our calculations, the radiolucent aluminum spacers between the radioopaque gold columns of our microslit collimator cut the dose within the microbeam down to a peak skin entrance dose rate of $27 \mathrm{~Gy} \mathrm{~s}^{-1} \mathrm{~mA}^{-1}$. The desired irradiation dose is obtained by selecting the speed of the vertically translated target through the beam.

\section{EXPERIMENTAL SETUP}

The Synchrotron Radiation x-ray beam is produced by bunches of $6 \mathrm{GeV}$ electrons circulating in an 844 metercircumference evacuated ring with an orbit time of $2.82 \mathrm{mi}-$ croseconds. The maximum resultant ring current of $200 \mathrm{~mA}$ has a typical decay time of 50 hours. For the dose measurements described below, only one bunch of electrons is used to minimize the dose rate to the detector. The ring current is a linear dose-scaling factor in MRT experiments. For our measurements, the maximum current was $20 \mathrm{~mA}$ with a typical lifetime of 6 hours. The beam in the first hutch of the ID17 beamline is spatially fractionated into microbeams at a distance of $33 \mathrm{~m}$ from the wiggler source. A set of tungsten slits of various sizes can be installed on a 3 -axis stage behind the collimator, either to block scattered radiation (vertical height of the slits $=500 \mu \mathrm{m}$ ) or to select one of the many available $\sim 20-30 \mu \mathrm{m}$ wide microbeams. To measure the peak valley dose ratios (PVDR) at the sample position, the MOSFET detector was installed on the sample stage (3-axis goniometer) $1.00 \mathrm{~m}$ downstream from the collimator. An ionization chamber at the downstream end of the hutch was used for alignment.

The specimen is fixed to a computer-controlled stage that permits high-resolution rotation and translation of the target area with respect to the beam. The irradiation is performed by vertically scanning the target through the beam, so that preselection of the speed of vertical translation of the target allows one to irradiate the selected tissue area with precisely the desired dose. A fast shutter system ${ }^{20}$ synchronized with the speed of vertical translation permits one to specify the time that each element of tissue in a microslice is exposed to the beam. An acceleration and deceleration path outside the beam is calculated and automatically applied to ensure a perfectly constant movement of the target during radiation exposure. A total irradiation field $10 \mathrm{~mm}$ wide and $10 \mathrm{~mm}$ high was chosen for this study. The minimum opening time of ID17's fast shutter is $30 \mathrm{~ms}$. Whenever a measurement of the exact opening time is required, a radiation-shielded photodiode is connected to an oscilloscope at the end of the beam path.

There exists no commercial device to measure the dose in the center of a several hundred $\mu \mathrm{m}$ wide "valley" or in the 


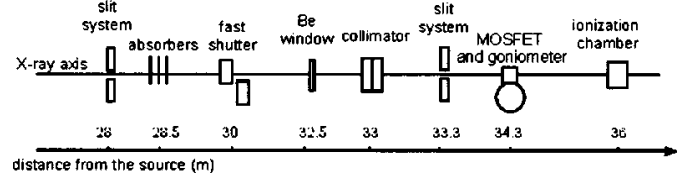

FIG. 2. Schematic view of the experimental setup.

midplane of a several tens of $\mu \mathrm{m}$ wide microslice of tissue, i.e., in the "peak." We now present dose measurements of valley and peak doses using a MOSFET-type detector operated in an "edge-on" mode. The current MRT program at the ESRF medical beamline ID17 uses a filtered, "white," broad-spectrum synchrotron radiation wiggler-generated beam with a critical energy of $38.1 \mathrm{keV}$, corresponding to a wiggler gap of $24.8 \mathrm{~mm}$. Our custom-made Archer-type multislit collimator ${ }^{21}$ can produce up to 80 planar microbeams of roughly uniform widths adjustable up to $50 \mu \mathrm{m}$ in width with an approximately uniform peak-to-peak interval of $\sim 210 \mu \mathrm{m}$. For this study the typical size of an array of microbeams is $10 \mathrm{~mm}$ wide and $500 \mu \mathrm{m}$ high, which typically irradiates a surface of $1 \mathrm{~cm}^{2}$ by vertical translation of the target through the beam. This corresponds to about 48 parallel, $\sim 25 \mu \mathrm{m}$ wide microbeams. Narrowing the width of the array makes a fairly homogeneous photon flux possible, thus assuring an equivalent peak dose from each microbeam. (See Fig. 2.)

\section{MATERIAL AND INSTRUMENTATION}

The actual MOSFET detector consists of four individual chips, which are mounted in a $12 \mathrm{~mm}$ thick housing (Fig. 3). The upper part of this encapsulation can be removed to acquire measurements without the PMMA encapsulation. The part of the support has a labyrinth design with an airtight fit; it also ensures that the central microbeam chosen for the measurements impinges onto an uninterrupted smooth surface a few millimeters from the joint where the two plastic pieces are screwed together. The measurements, called "free in air" in Table I, used a bare chip surrounded by a $300 \mu \mathrm{m}$ thick epoxy bulb and a small air gap of about $200 \mu \mathrm{m}$.

Measurements described in this article were performed using the on-line MOSFET readout system. ${ }^{22}$ The MOSFET detector is well known as a dosimetric detector. ${ }^{23-25}$ The

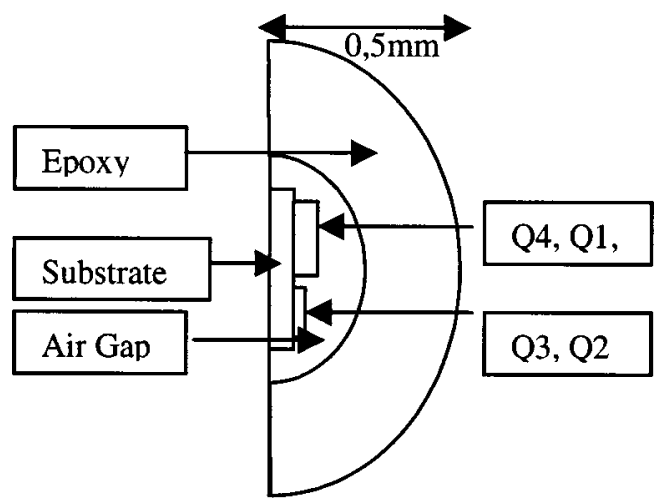

FIG. 3. Schematic diagram of MOSFET as the beam sees the detector.
TABLE I. Calibration results in terms of voltage shift per absorbed dose.

\begin{tabular}{lcc}
\hline \hline Free in air & $\begin{array}{c}\text { Behind } 3 \mathrm{~mm} \\
\text { of PMMA }\end{array}$ & $\begin{array}{c}\text { Behind } 1.2 \mathrm{~cm} \\
\text { of PMMA }\end{array}$ \\
\hline $62.64 \mathrm{mV} / \mathrm{Gy}$ & $68.75 \mathrm{mV} / \mathrm{Gy}$ & $63.56 \mathrm{mV} / \mathrm{Gy}$ \\
$\pm 4 \%$ & $\pm 4 \%$ & $\pm 4 \%$ \\
\hline \hline
\end{tabular}

particular MOSFET used in our experiments was the REM RADFET. $^{22}$ The RADFET consists of two pairs of $p$-channel MOSFETs with different gate oxide thicknesses $(\sim 0.13$ and $\sim 1 \mu \mathrm{m})$. The details of the topology and layout of the RADFET chip are described in Fig. 4.

\section{PACKAGING OF THE MOSFET DETECTOR FOR MICROBEAM EXPERIMENTS}

Our microbeam dosimetry approach is based on a quadrupole x-ray detector. Figure 4 shows a scanning electron microscope (SEM) image of the bare chip and the direction of the x-ray beam. This is the REM type TOT502 RADFET, a quadruple MOSFET chip with dimensions $1 \times 1 \times 0.5 \mathrm{~mm}^{3}$. Two MOSFETs, Q1 and Q4 (Type R), have gate oxide thickness values of about $0.9 \mu \mathrm{m}$ and two others, Q2 and Q3 (Type K), have thickness values of about $0.15 \mu \mathrm{m}$. The dosimeter sensors for this experiment were selected for preirradiation stability. The chip was encapsulated in opaque epoxy. On the axis of the microbeam array, the thickness of epoxy was less than $2 \mathrm{~mm}$.

For this experiment, chip carrier CC3 was used. This is a thin glass-epoxy carrier ("chip-on-board" technology) with 14 pins, which will connect with a standard DIL socket; the sensor is cantilevered so that a buildup cap can be placed around the chip. The normal built-in encapsulation is a hemisphere of opaque epoxy resin. The package was mounted in "edge-on" mode on a movable, computer-controlled, stage in the experimental hall. For high-energy photons, the sensor region was mounted inside a small block of PMMA $9 \times 9 \times 12$ $\mathrm{mm}^{3}$ - a "buildup cap" which created electronic equilbrium. The R-type RADFETs were irradiated under a $+5 \mathrm{~V}$ gate bias and the K-type under $\mathrm{a}+12 \mathrm{~V}$ gate bias. These irradia-

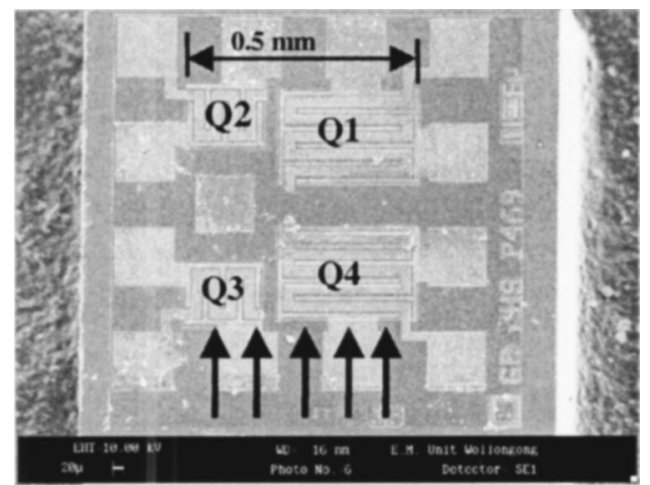

FIG. 4. Scanning electron microscopy (SEM) photograph of the REM TOT500 RADFET chip, showing the four RADFETs Q1-Q4. The "edgeon" geometry of the radiation beam for the experiments described in this article is shown impinging on the edges of Q3 and Q4. 
tion bias values were found to give the best linearity of threshold voltage versus dose. As the response curve rolls off slightly with accumulated dose, linearity was checked under different radiation pulse duration times.

For surface dose measurements, the center of the sensitive element of the "edge-on" RADFET chip was located at the center of the surface of the $\left(10 \times 10 \times 10 \mathrm{~cm}^{3}\right)$ PMMA phantom. The specially developed reader for pulsed beam applications allowed the readout of the MOSFET detector immediately after each exposure via a TTL pulse, generated by the beam shutter control computer, to trigger the readout system. All measurements were performed in an environment maintained at a temperature of $20^{\circ} \mathrm{C}$ controlled to within $0.5^{\circ} \mathrm{C}$.

The data presented in this report are based on parameters used for MRT. The calibration of the detectors was done under identical conditions to the experimental irradiations. The dose in front of the multislit collimator is known and cross calibrated with other detectors, such as ionization chambers and Gafchromic films. The two MOSFET R-type gate oxides have a size of $270 \times 180 \mu \mathrm{m}^{2}$ and a thickness of $1 \mu \mathrm{m}$, while the $0.15 \mu \mathrm{m}$ thick K-type chips cover an area of $120 \times 150 \mu^{2}$. The higher sensitive R-type chips were mainly used for alignment, since in this configuration the $\mathrm{x}$-ray flux can be reduced by opening the wiggler gap. Absolute dosimetric data were taken with less sensitive K-type chips to allow measurements at closed gap $(24.8 \mathrm{~mm})$. At a minimum dose per exposure of $8 \mathrm{~Gy}$, considering the necessity to do whole scans for the correct alignment, only a few measurements were possible in the peak, since the REM RADFET detector are saturated after about $400 \mathrm{~Gy}$. They can be replaced by the same detector type, but careful characterization of the new alignment takes several hours.

The chips lying in the used geometrical configuration have proven to be extremely useful for the correct alignment of the sensor with respect to the beam. During the alignment phase, the shift between the peaks of the scanned profiles of two chips on top of each other (with the observer looking downstream in beam direction), and those two chips in front and in the back allow calculation of the misalignment and their correction with pitch, yaw, and roll movements of the goniometer.

\section{DETECTOR LINEARITY}

The MOSFET detectors were used in active mode, with a bias on the gate to reduce the nonlinearity of the dose response for all measurements. For peak-to-valley dose measurements a $\sim 0.1 \mu \mathrm{m}$ gate oxide thickness and gate bias of $+12 \mathrm{~V}$ yielded $\sim 60 \mathrm{mV} / \mathrm{Gy}$ and was dose rate-independent due to the high internal electric field in the oxide under these conditions. The maximum dose rate for these experiments was $\sim 10^{2} \mathrm{~Gy} \mathrm{~s}^{-1}$. Dose linearity was checked regularly by repeating measurements of the increment of the threshold voltage shifts before and after groups of dose measurements.

\section{CALIBRATION RESULTS}

In order to calibrate the MOSFET detector, we calculated and measured the photon flux ${ }^{16-19}$ in front of the multislit collimator giving a regular dose for a homogeneous surface of $1 \mathrm{~cm}^{2}$. The MOSFET detector was calibrated in this homogeneous field with a known photon flux behind different thicknesses of PMMA. Three different geometry conditions were used within a homogeneous photon flux upstream from the multislit collimator, identical to the ones used for our dose measurements with calibrated Gafchromic films, i.e., equally done at the surface and behind $3 \mathrm{~mm}$ of PMMA. These irradiations can be done independently, placing either the MOSFETs or the Gafchromic films at exactly the same place behind identical PMMA thicknesses on the goniometer; the dose control is perfect reproducible by the software controlled exposure time and speed of the goniometer, which takes automatically the current of the machine into account to deliver the desired dose. The data presented in Table I represent the change in the response of the K-type MOSFETs per one gray delivered at the surface of the phantom. This is a reproducible known dose value measured by Gafchromic film dosimeters, read out with a photo spectrometer (U-2000 UV/Vis) at the ESRF. The response of the Gafchromic films above $50 \mathrm{keV}$ is nearly energy independent, which makes it a very adequate dosimeter for our spectrum. The films are calibrated with a Cobalt 60 source [MDS/Nordion type Gammacell 220 (ID34) of the service de Metrologie Habilite, Aerial, Schiltigheim, France]. Calibration curves using an x-ray generator are as well obtained at an energy around $79 \mathrm{keV}$ from the CEA Institute Grenoble, France.

When we apply the obtained calibration values in front of the multislit collimator to convert our voltage shifts into a peak dose, we underestimate the real dose in the peaks due to the slightly harder spectrum after the multislit collimator, which partially explains the difference between measured and calculated values in Fig. 6. These corrections will be the subject of further studies. Far more important are the PVDRs, which are barely affected by these calibration errors, even when $1 \mu \mathrm{m}$ resolution is required. The results of these calibrated MOSFET detectors are averaged for four different exposures per chip and shown in Table I.

\section{CHARACTERIZATION OF MICROBEAMS}

The multislit collimator ${ }^{21}$ used at the ESRF consists of two stacks of gold foils (high $Z$ ) reducing the photon flux by 99.99\% and low $Z$ aluminum foils reducing the photon flux only by $30 \%$. These stacks of foils can be moved parallel to each other with respect to the beam, resulting in arrays of microplanar beams (microbeams) having a width which can be set in the nominal interval of 5-50 $\mu \mathrm{m}$, and with a fixed peak-to-peak interval of $\sim 210 \mu \mathrm{m}$. During the manufacture of the collimator, significant variations in curvature and thickness of the gold and aluminum foils were introduced. In addition to these limitations, the intrinsic divergence of the wiggler source introduces further variations in every single microbeam width. In the case discussed here, where the average FWHM of the microbeams width was $25 \mu \mathrm{m}$, the variation range of their FWHM was 19-39 $\mu \mathrm{m}$. However, the two central microbeams used for our dose measurements measure (25.15 \pm 0.01$) \mu \mathrm{m}$ in FWHM (Fig. 5). A standard 


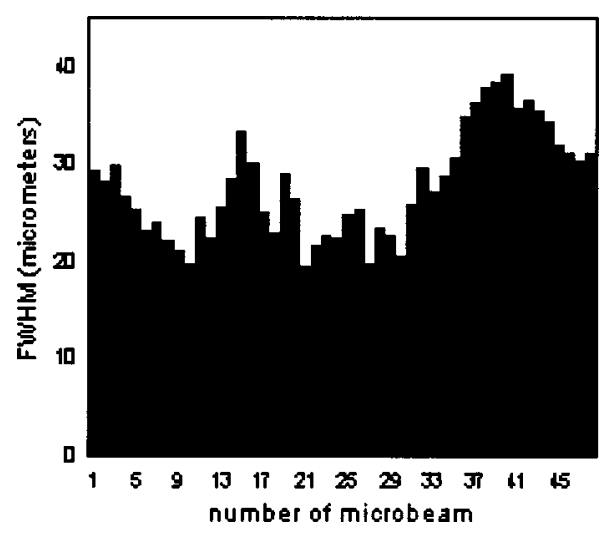

FIG. 5. The measured FWHM values of the microbeams. The average FWHM for the two central microbeams is $(25.15 \pm 0.01) \mu \mathrm{m}$.

alignment procedure for the collimator is applied to ensure an optimal production of microbeams, aiming at the ideal case of the gold parts not cutting the edges of a microbeam. In order to characterize the individual microbeams, a scan using polished tungsten slits with an aperture width of $5 \mu \mathrm{m}$ and a $1 \mu \mathrm{m}$ step size was done to measure the FWHM of each beam.

\section{RESULTS}

The substantial fluctuation of $\pm 30 \%$ in the FWHM of the peaks is probably an important factor in understanding differences between our measurements and the calculations made under the assumption of 48 perfectly planar microbeams with a $25 \mu \mathrm{m}$ FWHM each. The error for PVDRs associated herewith was estimated to be only $2 \%$. The lowest PVDRs are located at the vertical center of all microbeams, which corresponds therefore radiobiologically to the most crucial area and our focus of interest in this study.

Figures 6 and 7 show peak and valley doses measured on peak No. 25 and the valley dose between peak 25 and peak No. 26. These were the only neighboring microbeams with 25.1 and, respectively $25.2 \mu \mathrm{m}$ FWHM in the middle of the field. The corresponding PVDRs of these central beams are illustrated in Fig. 8. The data points are averaged for at least

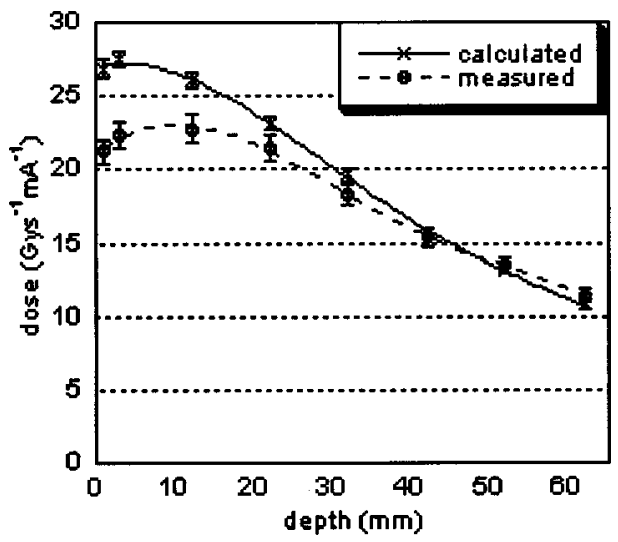

FIG. 6. Measured and calculated peak doses at different depths of a 10 $\times 10 \times 10 \mathrm{~cm}^{3}$ PMMA phantom.

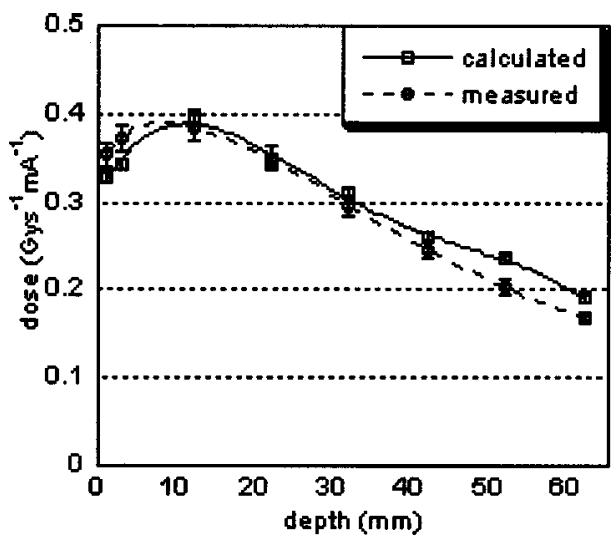

FIG. 7. Measured and calculated valley dose at different depths of a 10 $\times 10 \times 10 \mathrm{~cm}^{3}$ PMMA phantom.

two independent measurements, not necessarily with the same absorbed dose. They are scaled for the current in the storage ring and the measured exposure time, which are both linear factors.

Figure 7 shows a discrepancy between the simulated and measured relative dose in the depth-dose profile at depths greater than $12 \mathrm{~mm}$ (up to $60 \mathrm{~mm}$ ). The reason for this discrepancy can be explained by the different energy response of the MOSFET due to the change of the microbeam energy spectrum relative to the spectrum used for the calibration. The energy response of the MOSFET detector for x-ray fields has been addressed previously. ${ }^{26,27}$ In Fig. 7 no such discrepancies exist due to the fact that the valley dose is made up from scattered components from the primary beams and therefore the change in energy spectrum is less significant.

From the graphs of the measured data points in Figs. 6 and 7 we can apply an average PVDR of about 60 between the absorbed dose in the peak and in the valley at identical depth values. Since this PVDR is radiobiologically important, Fig. 8 visualizes that a PVDR of about 60 results in a good match of both curves measured by the MOSFET detector.

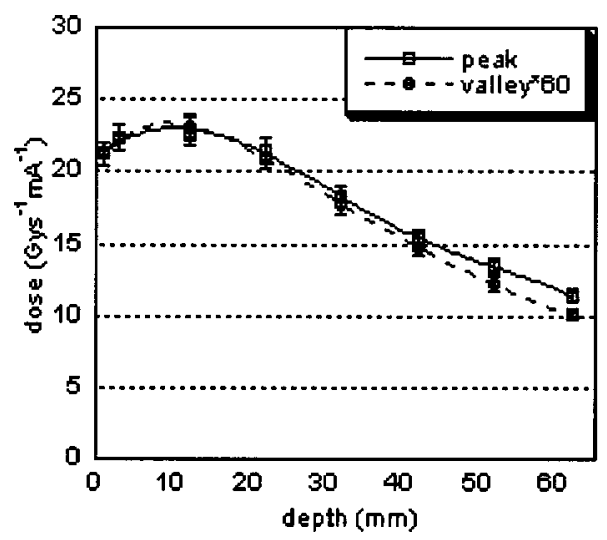

FIG. 8. Valley doses (squares) at different depths multiplied by a factor of 60 overlapped with peak doses (triangle) at identical depth positions. 


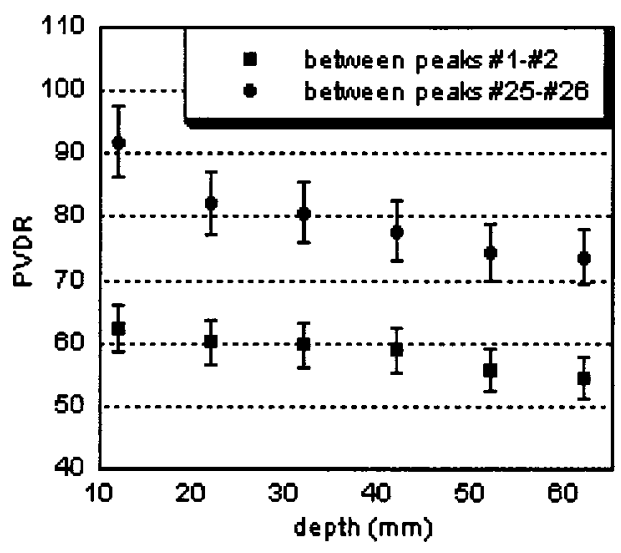

FIG. 9. Peak-valley ratios versus depth between central and outer peaks.

Since the decrease in dose versus depth for the peak dose and the valley dose is different, the curves show a different slope, indicating a more rapid decrease of the photons responsible for the dose in the microbeams, the reason for the peak-valley ratios decreasing in depth.

The PVDRs do not only depend on the position in depth, the size and number of the microbeams and the phantom size, etc., but as well on the location within the field, since the valley dose is more significant filled up in the center of the microbeam field due to the overlapping tails of each individual microbeam. For this reason, the PVDR in the center is the lowest and most critical. To verify this trend, we did measure the peak dose in peak N.1 and the valley dose between peak N.1 and peak N.2. The values reported in Fig. 9 show an expected increase of about 30\% in PVDRs in comparison with the curve obtained for dose measurements between peaks 25 and 26.

The theoretical Monte Carlo calculations consider the absorbed dose distribution around one single $25 \mu \mathrm{m}$ diameter planar microbeam of photons for a given energy spectrum in a water-equivalent phantom such as like PMMA. The peaks are placed $210 \mu \mathrm{m}$ apart and the multiple addition of the tails around the 48 microbeams then result in the valley dose. The tail of the most external microbeam extending towards the outside was measured by a scan using a small step size and long counting rates until the background noise detection had been reached. The result in Fig. 10 clearly shows that theoretical calculations should go as far out as $2 \mathrm{~cm}$ from the peak of one microbeam in order to take all scattering processes and related energy depositions for the correct valley dose calculation into account.

\section{DISCUSSION AND CONCLUSIONS}

The measurements using the MOSFET detector in "edgeon" mode demonstrated reproducible results matching well with theoretical calculations using the PSI-version of the GEANT Monte Carlo code. Absolute dose measurements in the peaks and in the valleys are about $20 \%$ less than the theoretically expected values. This gives credence to the conclusion that the calculation of the homogeneous photon flux through the rather sophisticated multislit collimator is not

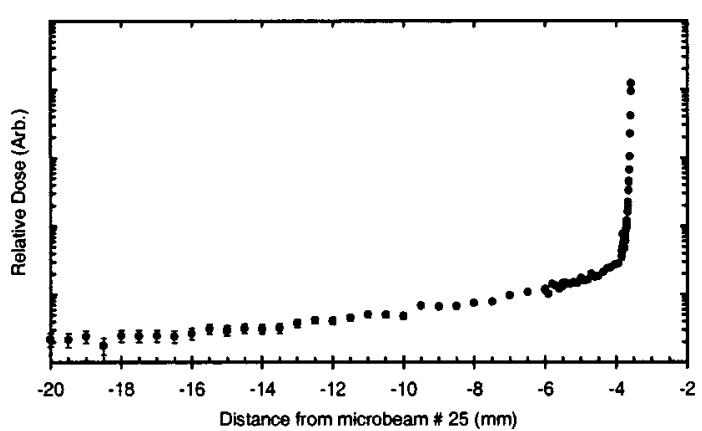

FIG. 10. Dose measurement approaching the first (most external) microbeam in the array.

realistic but this is difficult to achieve, since the exact geometry of the multislit collimator is an unknown. However, the important results of this work are the measured PVDRs confirming theoretical calculations within $5 \%$ over the entire range in depth. This is far more important, since for possible future therapeutic applications of MRT, the maximum peak dose is not the decisive factor; it must be ensured that the valley dose stays below a well-known tolerance dose value through the entire irradiated tissue volume in order to take advantage of the rapid repair of microscopic radiogenic lesions.

Our results demonstrating a good match of PVDRs 60 for the measured and calculated dose are obviously only a confirmation for this particular geometry. More studies are necessary to validate our Monte Carlo calculations when other important parameters such as field size, microbeam size, and size of the phantom vary.

Even to a tissue depth of $62 \mathrm{~mm}$, PVDRs only decrease from 91 to 65 and from 62 to 55 for the radial and central microbeams, respectively. This is very important considering a maximum depth of a tumor location to be reached where still sufficiently high enough PVDRs have to be attained.

\section{ACKNOWLEDGMENTS}

The authors thank the ID17 beamline team, in particular Thierry Brochard, for technical support. Christian Nemoz's and Gilles Berruyer's software assistance were particularly helpful. We also thank the teams involved in the MRT project, and commend the engineering workshops at the University of Wollongong and the ESRF for their skillful and timely fabrication of the phantoms. The authors thank Daniel Slatkin for his careful revision of the document.

\footnotetext{
${ }^{1}$ D. N. Slatkin, P. O. Spanne, F. A. Dilmanian, J.-O. Gebbers, and J. A. Laissue, "Subacute neuropathological effects of microplanar beams of x-rays from a synchrotron wiggler," Proc. Natl. Acad. Sci. U.S.A. 92, 8783-8787 (1995).

${ }^{2}$ D. N. Slatkin, P. O. Spanne, F. A. Dilmanian, and M. Sandborg, "Microbeam radiation therapy," Med. Phys. 19, 1395-1400 (1992).

${ }^{3}$ F. A. Dilmanian, G. M. Morris, G. Le Duc, X. Huang, B. Ren, T. Bacarian, J. C. Allen, J. Kalef-Ezra, I. Orion, E. M. Rosen, T. Sandhu, P. Sathé, X. Y. Wu, Z. Zhong, and H. L. Shivaprasad, "Response of avian embryonic brain to spatially segmented x-ray microbeams," Cell Mol. Biol. (Paris) 47, 485-494 (2001).
} 
${ }^{4}$ J. A. Laissue, G. Geiser, P. O. Spanne, F. A. Dilmanian, J.-O. Gebbers, M. Geiser, X. Y. Wu, M. S. Makar, P. L. Micca, M. M. Nawrocky, D. D. Joel, and D. N. Slatkin, Int. J. Cancer 78, 654-660 (1998).

${ }^{5}$ J. A. Laissue, N. Lyubimova, H.-P. Wagner, D. W. Archer, D. N. Slatkin, M. Di Michiel, C. Nemoz, M. Renier, E. Brauer, P. O. Spanne, J.-O. Gebbers, K. Dixon, and H. Blattmann, "Microbeam radiation therapy," Proc. SPIE 3770, 38-45 (1999).

${ }^{6}$ W. Thomlinson, P. Berkvens, G. Berruyer, B. Bertrand, H. Blattmann, E. Braeuer-Krisch, T. Brochard, A. M. Charvet, S. Corde, M. DiMichiel, H. Elleaume, F. Esteve, S. Fiedler, J. A. Laisseu, J. F. LeBas, G. Le Duc, N. Lyubimova, C. Nemoz, M. Renier, D. N. Slatkin, P. Spanne, and P. Suortti, "Research at the European Synchrotron Radiation Facility Medical Beamline," Cell Mol. Biol. (Paris) 46, 1053-1063 (2000).

${ }^{7}$ J. A. Laissue, H. Blattmann, M. DiMichiel, D. N. Slatkin, N. Lyubimova, R. Guzman, W. Zimmermann, T. Bley, P. Kircher, R. Stettler, R. Fatzer, A. Jaggy, H. M. Smilowitz, E. Brauer, A. Bravin, G. LeDuc, C. Nemoz, M. Renier, W. Thomlinson, J. Stepanek, and H. Wagner, "The Weanling Piglet cerebellum: A surrogate for tolerance to MRT (microbeam radiation therapy) in pediatric neuro-oncology," Proc. SPIE 4508, 65-73 (2001).

${ }^{8}$ I. Orion, A. Rozenfeld, F. A. Dilmanian, F. Telang, B. Ren, and Y. Namito, "Monte Carlo simulation of dose distributions from a synchrotronproduced microplanar beam array using the EGS4 code system," Phys. Med. Biol. 45, 2497-2508 (2000).

${ }^{9}$ J. Stepanek, H. Blattmann, J. A. Laissue, N. Lyubimova, M. DiMichiel, and D. N. Slatkin, "Physics study of microbeam radiation therapy with PSI-version of Monte-Carlo code Geant as a new computational tool," Med. Phys. 27, 1664-1675 (2000).

${ }^{10}$ D. Knespl, H. W. Reist, and J. Stepanek, "Particle track simulation in nanometer range for radiotherapy and radiation protection," Proceedings of SGSMP/SGBT-Jahrestagung 241, 1993.

${ }^{11}$ D. E. Cullen, S. T. Perkins, and J. A. Rathkopf, The 1989 Livermore Evaluated Photon Data Library (EPDL), Lawrence Livermore National Laboratory, Livermore, CA, UCRL-ID-103424, 1990.

${ }^{12}$ D. E. Cullen, M. H. Chen, J. H. Hubbell, S. T. Perkins, E. F. Plechaty, J. A. Rathkopf, and J. H. Scofield, Tables and graphs of photon-interaction cross sections from $10 \mathrm{eV}$ to $100 \mathrm{GeV}$ derived from LLNL Evaluated Nuclear Data Library (ENDL), Lawrence Livermore National Laboratory, Livermore, CA, UCRL-50400, Vol. 6, Rev. 4, 1989.

${ }^{13}$ S. T. Perkins, D. E. Cullen, and S. M. Seltzer, Tables and graphs of electron-interaction cross sections from $10 \mathrm{eV}$ to $100 \mathrm{GeV}$ derived from
LLNL Evaluated Nuclear Data Library (ENDL) Z $=1-100$, Lawrence Livermore National Laboratory, Livermore, CA, UCRL-50400, Vol. 31, 1991.

${ }^{14}$ S. T. Perkins, D. E. Cullen, M. H. Chen, J. H. Hubbell, and A. Rathkopf, Tables and graphs of atomic subshell and relation data derived from LLNL Evaluated Atomic Data Library (EADL), Lawrence Livermore National Laboratory, Livermore, CA, UCRL-50400, Vol. 30, 1991.

${ }^{15}$ W. R. Nelson, H. Hyrayama, and D. W. O. Rogers, "The EGS4 code system," SLAC Report 265, 1985.

${ }^{16}$ M. Sánchez del Río and R. J. Dejus, "XOP: Recent developments," Proc. SPIE 3448 (1998).

${ }^{17}$ M. Sánchez del Rio and R. J. Dejus, "XOP: A multiplatform graphical user interface for synchrotron radiation spectral and optics calculations," Proc. SPIE 3152, 148-157 (1997).

${ }^{18}$ R. J. Dejus and M. Sánchez del Rio, "XOP: A graphical user interface for spectral calculations and x-ray optics utilities," Rev. Sci. Instrum. 67, 3356 (1996), abstract.

${ }^{19}$ V. Honkimaeki and P. Suortti, "Whole-pattern fitting in energy-dispersive powder diffraction," J. Appl. Crystallogr. 25, 97-104 (1992).

${ }^{20}$ M. Renier, T. Brochard, C. Nemoz, and W. Thomlinson, "A white-beam fast-shutter for microbeam radiation therapy at the ESRF," Nucl. Instrum. Methods A 479, 656-660 (2002).

${ }^{21}$ D. W. Archer, Collimator for Producing an array of microbeams, U.S. Patent No. 5771270; June 23, 1998.

${ }^{22}$ A. B. Rosenfeld, M. Lerch, T. Kron, E. Brauer-Krisch, A. Bravin, and A. Holmes-Siedle, "Feasibility study of on-line, high spatial resolution MOSFET dosimetry in static and pulsed x-ray radiation fields," IEEE Trans. Nucl. Sci. 48, 2061-2068 (2001).

${ }^{23}$ A. Holmes-Siedle and C. Adams, "RADFETs: A review of the use of metal-oxide-silicon devices as interfration dosimeters," Radiat. Phys. Chem. 28, 235-244 (1986).

${ }^{24}$ A. B. Rosenfeld, G. I. Kaplan, T. Kron, B. J. Allen, A. Dilmanian, I. Orion, B. Ren, M. Lerch, and A. Holmes-Siedle, "MOSFET dosimetry of x-ray microbeams," IEEE Trans. Nucl. Sci. NS-46, N6, 1774-1780 (1999).

${ }^{25}$ G. Kaplan, A. B. Rosenfeld, B. Allen, T. Kron, and A. Holmes-Siedle, "Improved spatial resolution by MOSFET dosimetry of an X-ray microbeam," Med. Phys. 27, 239-244 (2000).

${ }^{26}$ A. B. Rosenfeld, M. G. Carolan, B. J. Allen, and V. I. Khivrich, "MOSFET Dosimeters: role of encapsulation in mixed gamma-neutron and megavoltage x-ray fields,” IEEE Trans. Nucl. Sci. NS-42, 1870-1877 (1995).

${ }^{27}$ C. R. Edwards, S. Green, J. E. Palethorpe, and P. J. Mountford, "The response of a MOSFET, p-type semiconductor and LiF TLD to quasimonoenergetic x-rays," Phys. Med. Biol. 42, 2383-2391 (1997). 